RESEARCH REPORT

\title{
"Unequal opportunity": neighbourhood disadvantage and the chance to buy illegal drugs
}

\author{
C L Storr, C-Y Chen, J C Anthony
}

J Epidemiol Community Health 2004;58:231-237. doi: 10.1136/jech.2003.007575

See end of article for authors' affiliations

.....................

Correspondence to: Carla Storr, Department of Mental Health, Johns Hopkins Bloomberg School of Public Health, $624 \mathrm{~N}$ Broadway, 8th Floor, Baltimore, MD 21205, USA; cstorr@ihu.edu

Accepted for publication 9 September 2003
Study objectives: This study investigates whether subgroups of people living in disadvantaged neighbourhoods may be more likely to come into contact with drug dealers as compared with persons living in more advantaged areas, with due attention to male-female and race-ethnicity differences.

Design: Standardised survey data collected using stratified, multistage area probability sampling. Setting: United States of America, 1998.

Participants: Nationally representative sample of household residents age 12 or older $(n=25500)$.

Main results: Evidence supports an inference that women are less likely to be approached by someone selling illegal drugs. The study found no more than modest and generally null racial and ethnicity differences, even for residents living within socially disadvantaged neighbourhoods, where chances to buy illegal drugs are found to be more common.

Conclusions: Limitations of survey data always merit attention, but this study evidence lends support to the inference that physical and social characteristics of a neighbourhood can set the stage for opportunities to become involved with drugs.
1 this research, we draw upon data from a cross sectional survey, with a nationally representative probability sample of household residents in the United States of America, investigating population level variation in the occurrence of a "drug purchase opportunity," with coverage of urban-rural differences and variation across US census regions. This study also includes a more focused inquiry on variation in relation to a gradient of neighbourhood level social disadvantage. With focus on a very early stage of drug involvement, population survey questions about drug purchase opportunity do not ask respondents to admit an illegal behaviour. As such, these questions may be less sensitive to measurement error, as compared with questions about recent illegal drug use. ${ }^{12}$ Assessment of a recent drug purchase opportunity is accomplished by asking respondents one or more standardised questions of the following form: "In the past 30 days, has anyone approached you to sell an illegal drug?" For most people, it is neither a particularly sensitive nor embarrassing experience to have encountered this form of drug purchase opportunity. In contrast, actual illegal drug use may be under-reported except when the respondent feels that confidentiality safeguards are complete. ${ }^{3}$

Our current focus on drug purchase opportunities is consistent with a more general orientation to the earliest stages of drug involvement. The trajectory of early drug involvement includes opportunities to try an illegal drug, often via peer to peer sharing without remuneration. ${ }^{4-7}$ However, as revealed in recent national surveys and in this research report, each month, noteworthy proportions of United States residents experience the chance to buy drugs from an illegal drug dealer. ${ }^{1}$ Our focus on gradients of neighbourhood level social disadvantage and drug purchase opportunities builds from prior research on drug exposure opportunities in general, which has been found to occur more often in socially disadvantaged neighbourhoods, though in a non-linear fashion as compared with a linear and monotonic gradient. $^{8-9}$ For this reason, to be consistent with the approach used in prior research on drug exposure opportunity, we expressed occurrence of drug purchase opportunities as a function of a gradient in the level of local area disadvantage.

These focal points represent an appreciation of several important challenges in epidemiological research on drug involvement-namely, to understand the larger community contexts that might influence illegal drug use, to illuminate urban-rural differences and regional variations, and to clarify the variations in drug involvement that may exist within each community. For example, visibility of drug trafficking and related crime in disadvantaged inner city communities does not necessarily mean that all residents of these disadvantaged communities are using illegal drugs. ${ }^{11}$ None the less, even though no community in the United States seems to be immune from illegal drug use, drug trafficking activities often appear to be more well established and are more visible in disadvantaged urban neighbourhoods. Drug trafficking can offer economic advantages that are difficult to ignore in contexts of general impoverishment and an especially constrained set of pathways and access to material wealth or social status within a generally affluent society. ${ }^{12}$ In addition, social capital and social cohesion create contexts that should dampen participation in illegal behaviours and unhealthy developmental experiences, including drug purchase opportunities. ${ }^{13}$

Social controls that influence drug trafficking directly or indirectly may include efforts to increase police presence, to reduce disorderly conduct, fighting, and violence, and to discourage violations of broadly shared social norms in general. In addition, efforts to reduce graffiti and litter and to promote owner occupied housing may improve the physical environment of the neighbourhood while promoting social cohesion and rule abidance. ${ }^{14}$ Reno and colleagues, among others, have found that people are more likely to litter in local area environments that already have been trashed. ${ }^{15}$ The occurrence of gonorrhoea is increased in neighbourhoods with deteriorated physical conditions, independent of poverty. ${ }^{16}$ The occurrence of violent crime is linked to a higher density of beverage alcohol outlets-the prime locus for alcohol purchase opportunities in the United States. ${ }^{17}$ 
Table 1 Sample description and estimated occurrence of a recent drug purchase opportunity, in relation to respondent characteristics: data from US National Household Survey on Drug Abuse, NHSDA 1998

\begin{tabular}{|c|c|c|c|c|c|}
\hline & Number & Estimated $\uparrow \%$ & Odds ratio & $95 \% \mathrm{Cl}$ & p Value \\
\hline \multicolumn{6}{|l|}{ Sex } \\
\hline Female & 14239 & 4.1 & 1.0 & & \\
\hline Male & 11261 & 8.2 & 2.1 & (1.8 to 2.4$)$ & $<0.001$ \\
\hline \multicolumn{6}{|l|}{ Age } \\
\hline 18 or older & 18722 & 5.3 & 1.0 & & \\
\hline $12-17$ years & 6778 & 13.7 & 2.8 & (2.4 to 3.3$)$ & $<0.001$ \\
\hline \multicolumn{6}{|l|}{ Race } \\
\hline White, non-Hispanic & 11709 & 5.2 & 1.0 & & \\
\hline Black, non-Hispanic & 5815 & 9.4 & 1.9 & (1.5 to 2.3 ) & $<0.001$ \\
\hline Hispanic & 6795 & 9.3 & 1.9 & (1.6 to 2.2$)$ & $<0.001$ \\
\hline Other, non-Hispanic & 1181 & 4.1 & 0.8 & (0.5 to 1.1$)$ & 0.20 \\
\hline \multicolumn{6}{|l|}{ Education } \\
\hline$>12$ th grade & 7783 & 4.1 & 1.0 & & \\
\hline High school or less & 10939 & 7.7 & 1.9 & (1.6 to 2.4$)$ & $<0.001$ \\
\hline \multicolumn{6}{|l|}{ Census division } \\
\hline North East and Mid Atlantic & 3121 & 5.4 & 1.0 & & \\
\hline North Central & 3680 & 5.9 & 1.1 & $(0.8$ to 1.6$)$ & 0.57 \\
\hline South Atlantic & 3959 & 6.4 & 1.2 & (0.8 to 1.7 ) & 0.30 \\
\hline South Central & 4545 & 6.4 & 1.2 & $(0.9$ to 1.7$)$ & 0.28 \\
\hline Mountain & 4779 & 5.2 & 1.0 & $(0.6$ to 1.4$)$ & 0.85 \\
\hline Pacific & 5416 & 6.7 & 1.3 & $(0.9$ to 1.7$)$ & 0.14 \\
\hline \multicolumn{6}{|l|}{ Urbanicity } \\
\hline Rural & 3579 & 4.5 & 1.0 & & \\
\hline Urban & 21930 & 6.5 & 1.5 & (1.2 to 1.8$)$ & $<0.001$ \\
\hline \multicolumn{6}{|l|}{ Past year drug use* } \\
\hline No & 22109 & 3.4 & 1.0 & & \\
\hline Yes & 2797 & 28.5 & 11.3 & (9.7 to 13.3 ) & $<0.001$ \\
\hline \multicolumn{6}{|c|}{$\begin{array}{l}\text { Quartile of neighbourhood disadvantage as rated by others living in the same area segment as } \\
\text { the respondent }\end{array}$} \\
\hline Least disadvantaged & 6314 & 4.2 & 1.0 & & \\
\hline Quartile 2 & 6315 & 6.1 & 1.5 & (1.2 to 1.8 ) & $<0.001$ \\
\hline Quartile 3 & 6315 & 7.4 & 1.8 & (1.5 to 2.2 ) & $<0.001$ \\
\hline Most disadvantaged & 6315 & 10.3 & 2.6 & (2.1 to 3.3 ) & $<0.001$ \\
\hline
\end{tabular}

*Any extra-medical or illegal drug use in past year including marijuana, cocaine and crack, hallucinogens, heroin, and inhalants. †Estimated prevalence (\%), after sample weighting and post-stratification adjustment. Estimated

$\mathrm{OR}=$ odds ratio from contingency table analysis, no statistical adjustment for covariates.

Against this background of prior theory and evidence, ${ }^{8}$ we posit that the event of a drug purchase opportunity is located within a larger macrosocial context. The macrosocial context may determine whether a person will experience a drug purchase opportunity at any given point in time. Based on this conceptual model, we are expressing a person's experience of a recent drug purchase opportunity as a function of the more macrosocial context of social disadvantage. Notwithstanding the possibility that an accumulating number of individual drug opportunities will yield changes in the macrosocial context (for example, an increasing police presence), we do not think that any individual drug purchase opportunity, in general, will evoke a change in the larger macrosocial context in any direct fashion.

We acknowledge that our cross sectional study cannot disentangle social selection and social causation processes that might account for observed associations linking recent drug purchase opportunities back to levels of neighbourhood disadvantage. ${ }^{18-20}$ Moreover, the density of drug purchase opportunities at one point in time may have an indirect causal influence on later levels of neighbourhood disadvantage, although we know of no definitive evidence about this potential reciprocal influence. Likewise, no one has shown that a person's experience of a drug purchase opportunity near home induces a later more generalised and negative evaluation of the local neighbourhood.

In our investigation, anticipating variation within community contexts, we have looked for male-female differences of the type that others have found in past studies of drug exposure opportunities in general and drug purchase opportunities in particular. ${ }^{2521}$ By stratifying on levels within the gradient of neighbourhood disadvantage, we seek generalisable statements about male-female differences and race-ethnicity differences in the occurrence of drug purchase opportunities, holding constant the level of neighbourhood disadvantage. By stratifying to hold constant male-female and race-ethnicity differences, we seek generalisable statements about how occurrence of recent drug exposure opportunities might depend upon the gradient of neighbourhood disadvantage.

\section{METHODS}

\section{Study population}

Data under study are from the 1998 National Household Survey on Drug Abuse (NHSDA). ${ }^{22}$ For more than 20 years, NHSDA has provided cross sectional estimates on the prevalence and patterns of drug use in the United States. During these years a nationally representative sample of non-institutionalised civilians aged 12 or older has been drawn, using stratified, multistage area probability sampling. In 1998, as in other years, the first stage of the sampling started by selecting primary sampling units defined as metropolitan areas, counties, groups of counties, and cities. These primary sampling units are further divided into area segments approximating groups of housing units that closely equate to what we will refer to as neighbourhoods (for example, census tracts or block groups). Then, dwelling units within the area segments are sampled probabilistically. Finally, persons within occupied dwelling units are selected at random within pre-designated age groups. To provide more precise national estimates for subgroups, the NHSDA over-samples these groups (for 
example, adolescents and those in racial ethnic minority groups).

The survey sample in 1998 included 25500 people; table 1 shows the sample breakdown on several sociodemographic characteristics. More than half of the sample was female $(56 \%)$ and $46 \%$ of the sample was white, non-Hispanic. For youths aged $12-17$ years, a parent or legal guardian was given a description of the survey and participant consent was obtained in accordance with procedures approved by the institutional review board responsible for this research. The overall response rate was $77 \%$ for the 1998 survey.

\section{Data collection}

Data came from responses offered by each participant during an individualised assessment session, typically at or near the home of the participant. The NHSDA survey team extensively trains their interviewers to minimise interviewer variation and bias. Trained interviewers follow a structured format while administering standardised questions with almost exclusively pre-specified and pre-coded response categories and virtually no allowance for probing questions. Self administered answer sheets are used to constrain underreporting of sensitive issues. The result is an interview that leaves very little in the hands of the individual interviewer once assurances of confidentiality have been made, a private location for the interview has been secured, and issues of trust and rapport have been established.

\section{Measures}

\section{Neighbourhood disadvantage}

Participants were asked 11 standardised items about the physical environment of their neighbourhoods of residence (for example, abandoned buildings, graffiti) and about locally prevailing social conditions (for example, crime, family violence, helpfulness of people). These coded responses (for example, strongly agree $=1$, somewhat agree $=2$, somewhat disagree $=3$, and strongly disagree $=4$ ) were first summed for each person, labelled as the index person. A check for internal consistency using Cronbach's $\alpha$ was 0.82 , well above the 0.7 level advocated by Nunnally. ${ }^{23}$ Potential biases can arise when the same person in the same interview session has been asked questions about drug experiences and neighbourhood conditions. Therefore, instead of using each subject's responses to characterise neighbourhood conditions in the local area, we calculated a mean neighbourhood disadvantage score by drawing values from every other respondent living in the same neighbourhood, excluding the index person's response value. The sample contains 2627 area segments, with the number of people in them ranging from 1 to 51 (mean number of residents in a segment $=13$ ). Segments with only one interview respondent had to be dropped $(n=56)$. As measured for this study, the neighbourhood disadvantage score for each index person's neighbourhood is characterised by responses of other respondents living in the same area; the summary value for neighbourhood disadvantage is not the value reported by the index respondents themselves.

\section{Opportunities to use drugs}

In earlier years of NHSDA fieldwork, a person's drug exposure opportunity was assessed via standardised questions on age at first chance to try individual drugs such as marijuana and cocaine. ${ }^{5620}$ These questions were dropped in 1995 to make room for other assessment modules, but in 1998 an assessment of drug exposure opportunity was reintroduced via a single question about recent experience: "In the past 30 days, has anyone approached you to sell an illegal drug?" In this revised assessment, it might be expected that recall bias and retrospection error are constrained by a focus on drug related experiences in the month before the date of the assessment. Sociodemographic characteristics of the respondents and their use of illegal drugs (marijuana, cocaine and crack, hallucinogens, heroin, and inhalants) also were assessed via standardised survey items.

\section{Statistical analysis}

Procedures under Stata version 7.0 (Stata statistical software, TX, USA) were used to take into account the complex sampling design and weights. Preliminary contingency tables yielded estimates for the unadjusted association between neighbourhood disadvantage and having a recent opportunity to purchase drugs, as well as male-female and raceethnicity subgroup differences in the strength of association. For these analyses, the neighbourhood disadvantage score was standardised and index respondents were sorted into subgroups of disadvantage based on quartiles of the summary score calculated from responses made by the other respondents living in the same survey segment. Thereafter, the Stata multiple logistic regression command "svylogit" designed to handle special requirements of complex survey data was used to obtain weighted estimates while addressing possible confounding by individual characteristics that might influence a drug dealer to approach a person (for example, age). Additional models also adjusted for apparent differences between neighbourhood via education, urbanicity, and census regions. These estimates are not shown as they were not appreciably different from those found in models that adjusted for individual characteristics.

Table 2 Estimated odds ratios $(95 \% \mathrm{Cl})$ to index the strength of associations between neighbourhood disadvantage and being approached by someone selling drugs, NHSDA 1998

\begin{tabular}{|c|c|c|c|c|c|c|c|c|c|}
\hline & \multicolumn{3}{|c|}{ Overall } & \multicolumn{3}{|c|}{ Female } & \multicolumn{3}{|l|}{ Male } \\
\hline & $\%$ & aOR* $(95 \% \mathrm{Cl})$ & $\mathrm{p}$ Value & $\%$ & $\mathrm{aOR}+(95 \% \mathrm{Cl})$ & p Value & $\%$ & $\mathrm{aOR}+(95 \% \mathrm{Cl})$ & p Value \\
\hline Least disadvantaged & 4.2 & 1.0 & & 2.7 & 1.0 & & 5.8 & 1.0 & \\
\hline Quartile 2 & 6.1 & $1.3(1.0$ to 1.6$)$ & 0.02 & 3.9 & $1.3(1.0$ to 1.9$)$ & 0.07 & 8.3 & 1.3 (1.0 to 1.7$)$ & 0.07 \\
\hline Quartile 3 & 7.4 & $1.5(1.2$ to 1.9$)$ & $<.001$ & 4.4 & $1.5(1.0$ to 2.0$)$ & 0.03 & 10.5 & $1.5(1.1$ to 2.1$)$ & 0.01 \\
\hline \multirow[t]{3}{*}{ Most disadvantaged } & 10.3 & $2.2(1.7$ to 2.7$)$ & $<.001$ & 8.1 & $2.9(2.0$ to 4.4$)$ & $<0.001$ & 13.1 & $1.8(1.3$ to 2.4$)$ & $<0.001$ \\
\hline & & & & \multicolumn{3}{|c|}{ No illegal drug use in past year } & \multicolumn{3}{|c|}{ Illegal drug use in past year } \\
\hline & & & & $\%$ & $a O R^{*}(95 \% \mathrm{Cl})$ & p Value & $\%$ & $a O R^{*}(95 \% \mathrm{Cl})$ & p Value \\
\hline Least disadvantaged & & & & 2.2 & 1.0 & & 25.6 & 1.0 & \\
\hline Quartile 2 & & & & 3.5 & 1.4 (1.0 to 1.9$)$ & 0.06 & 27.7 & $1.1(0.8$ to 1.4$)$ & 0.68 \\
\hline Quartile 3 & & & & 4.3 & $1.4(1.1$ to 2.0$)$ & 0.02 & 27.8 & $1.1(0.8$ to 1.5$)$ & 0.57 \\
\hline Most disadvantaged & & & & 6.0 & $1.9(1.4$ to 2.6$)$ & $<0.001$ & 36.7 & 1.8 (1.2 to 2.7$)$ & 0.002 \\
\hline
\end{tabular}

aOR, adjusted odds ratio; * with multiple logistic regression adjustment for covariates: being male, race, and age; twith multiple logistic regression adjustment for covariates: race and age. 


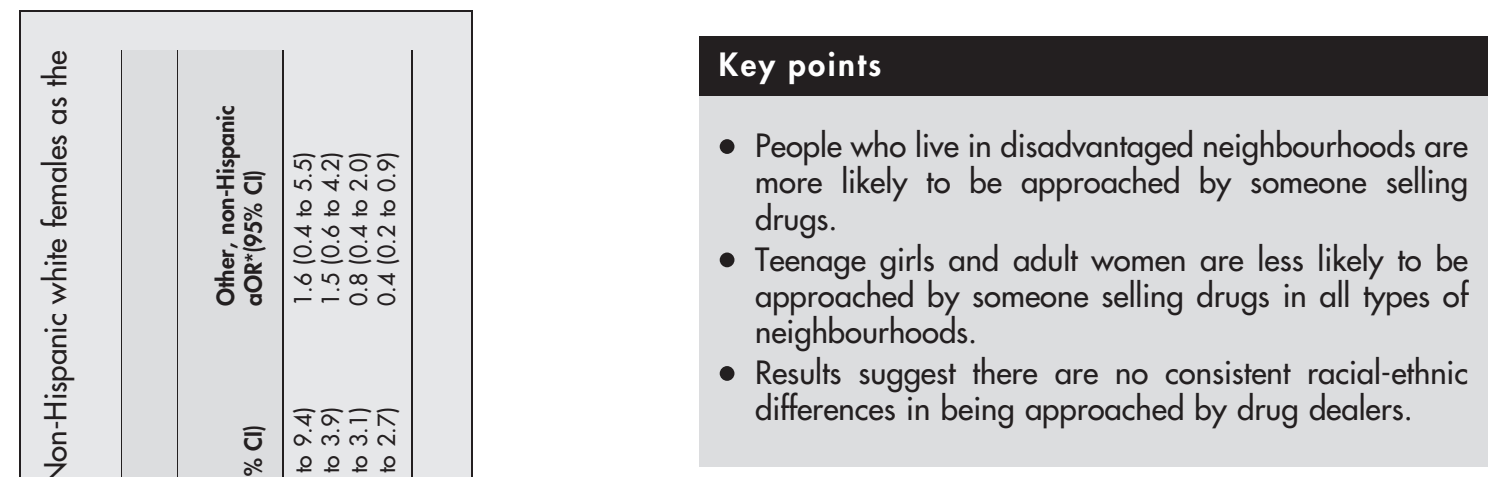

As a check on the study's statistical power to detect subgroup differences, we conducted a post hoc power analysis for the contrast between our smallest racial-ethnic minority group compared with the largest group. In all but "Other, non-Hispanic" male and female sub-analyses, the sample sizes are large enough to detect an odds ratio estimate of 1.5 (or 0.67 ) as significant at $\mathrm{p}<0.05$, with $\alpha$ set at 0.05 and $\beta$ at 0.20 .

\section{RESULTS}

Based on the survey estimates, during one month intervals in $1998,6 \%$ of the survey population had a recent drug purchase opportunity ( $95 \%$ confidence intervals, $\mathrm{CI}=5.6$ to 6.6 ). Drug purchase opportunity was not limited to people living in urban areas or to those living in disadvantaged neighbourhoods (table 1). Subgroups with greater opportunity to buy drugs are males, those with less than 12 years of schooling, youths age 12-17 years old, and those living in urban areas. There was no substantial variation in occurrence of drug purchase opportunities across the six US census regions under study.

Residents in the most disadvantaged neighbourhoods were an estimated 2.2 times more likely to have an opportunity to obtain drugs $(95 \% \mathrm{CI}=1.7$ to $2.7, \mathrm{p}<0.001)$, as compared with residents in the lowest quartile of neighbourhood disadvantage (our reference category).

A gradient of excess occurrence of drug purchase opportunity was present for the both sexes, and separately for males and females (table 2).

As one half of the people who indicated an opportunity to purchase a drug in the 30 days before the interview indicated that they had used drugs in the past year, we explored a possibility that the association between neighbourhood disadvantage and opportunity to buy drugs was explained by drug dealers seeking out drug users or users seeking out dealers. We found that people with illegal drug use in the year before the interview were substantially more likely to have been approached by someone selling drugs. However, about $3 \%$ of US residents with no recent drug use also reported being approached by someone selling drugs (table 1). As neighbourhood disadvantage increases, there is an increased occurrence for drug purchase opportunities among those who did not use illegal drugs in the past year, whereas there is virtually no increase in such opportunities for recently active drug users except in the most disadvantaged neighbourhoods (table 2). Residents living in the more disadvantaged neighbourhoods were more likely to have experienced a recent drug purchase opportunity without respect to their own illegal drug use.

In analyses stratified for level of neighbourhood disadvantage, there was a generally stable inverse association between the odds of being approached by a drug dealer and being a female. As shown in the first four columns of table 3, no matter what the level of neighbourhood disadvantage, nonHispanic white women, non-Hispanic black women, and 


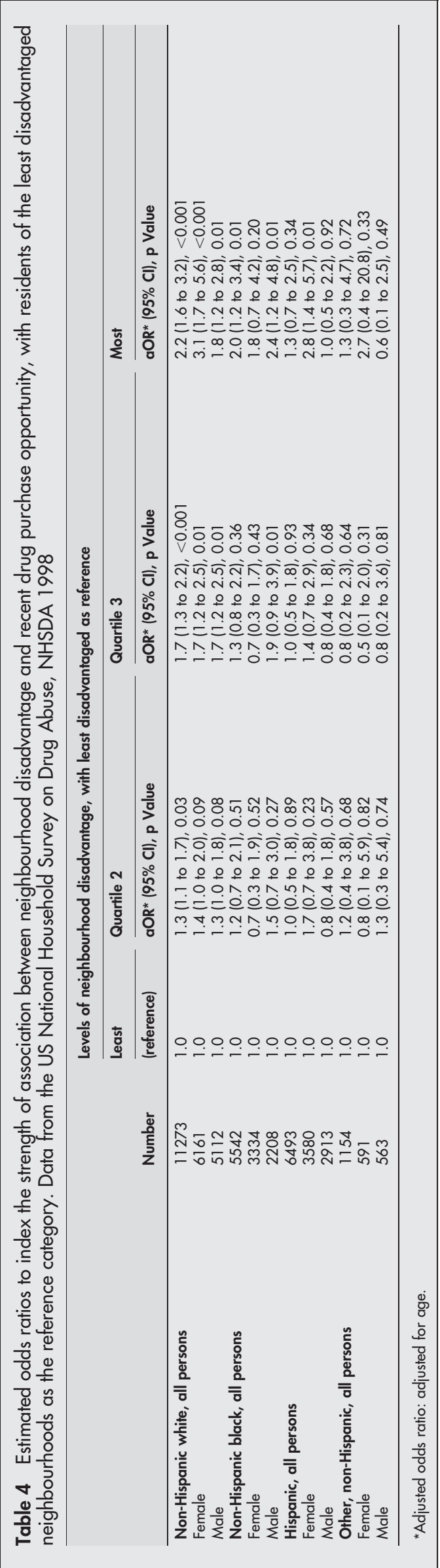

\section{Policy implications}

- To develop effective community level interventions an individual focused social psychology is not enough. Thinking ecologically, we must understand how various aspects of the social context (for example, neighbourhoods, schools, families, peers) work independently and jointly with one another.

- Programmes that target drug purchase opportunities while also changing the environment that supports the drug context might break cycles of passively experienced drug involvement, as well as active drug seeking behaviour, which otherwise perpetuate drug associated public health hazards.

- With the Human Genome Project, attention has shifted towards individual vulnerability or susceptibility traits. But with respect to drug use and dependence, the drug is a necessary "agent". The reservoirs of drug supply and vectors that convey drugs to vulnerable people also merit research focus, and a swing of the pendulum toward environmental contexts.

Hispanic women have roughly equivalent experience with recent drug purchase opportunities (for example, for nonHispanic black women compared with non-Hispanic white women in the most disadvantaged neighbourhoods: $\mathrm{aOR}=$ 1.1, $95 \% \mathrm{CI}=0.6$ to 1.9; for Hispanic women compared with non-Hispanic White women in the most disadvantaged neighbourhoods: aOR $=0.9,95 \% \mathrm{CI}=0.6$ to 1.6 ).

The male-female difference in odds of a recent drug purchase opportunity shows a very interesting pattern of subgroup variation that involves racial-ethnic minority status. Within neighbourhoods having the lowest levels of disadvantage (that is, least disadvantaged), in comparison with non-Hispanic white women, the non-Hispanic white men are an estimated 2.1 times more likely to have had a recent drug purchase opportunity ( $95 \% \mathrm{CI}=1.5$ to 2.9 ); nonHispanic black men are two times more likely to have had a recent drug purchase opportunity ( $95 \% \mathrm{CI}=1.2$ to 4.5 ), and Hispanic men are four times more likely to have had a recent drug purchase opportunity $(95 \% \mathrm{CI}=1.9$ to 9.4$)$. Within the other quartiles of neighbourhood disadvantage, a similar pattern of male-female difference can be seen, but the subgroup variation associated with minority status of the males is much less pronounced. For example, in the highest quartile of neighbourhood disadvantage (last row of table 3), with non-Hispanic white females as a reference category, we see roughly comparable odds ratio estimates for nonHispanic white males $(\mathrm{aOR}=1.3)$, non-Hispanic black males $(\mathrm{aOR}=2.1)$, and Hispanic males $(\mathrm{aOR}=1.6)$.

In summary, men are more likely to experience drug purchase opportunities, as compared with women, no matter what the level of neighbourhood disadvantage. With respect to women and occurrence of drug purchase opportunity, there is no subgroup variation involving race-ethnicity, no matter what level of neighbourhood disadvantage is considered. With respect to men and occurrence of drug purchase opportunity, men in historically disadvantaged racial-ethnic minority groups generally are more likely to have experienced drug purchase opportunities when level of neighbourhood disadvantage is low, but there is no subgroup variation involving race-ethnicity at the highest levels of neighbourhood disadvantage.

Table 4 presents evidence on gradients in the occurrence of recent drug purchase opportunity across levels of 
neighbourhood disadvantage, with stratification on malefemale and race-ethnicity status. For example, in the first row of table 4 , we see that for the non-Hispanic white population (males and females combined), the odds of a recent drug purchase opportunity are lowest at the lowest level of neighbourhood disadvantage, and become higher across quartiles of increasing neighbourhood disadvantage. However, increasing occurrence of drug purchase opportunities across levels of neighbourhood disadvantage is not seen for all male-female and race-ethnicity groups. Row by row, in comparisons of females of the same race-ethnicity subgroup, those residents in neighbourhoods with the greatest levels of disadvantage tended to be more likely to be approached by a drug dealer as compared with racially similar females living in less disadvantaged neighbourhoods. In race-ethnicity subgroups of men, this pattern is not so prominent.

\section{DISCUSSION}

These results, based on a nationally representative sample of household residents within the United States, tend to indicate that people who live in disadvantaged neighbourhoods are more likely to be approached by someone selling drugs, consistent with a general idea that it is easier for residents in disadvantaged neighbourhoods to obtain illegal drugs. Overall, about one half of those with an opportunity to buy drugs reported using illegal drugs in the year before the interview, but even so, an estimated $3 \%-4 \%$ of non-users had recently experienced a drug purchase opportunity. The association between neighbourhood disadvantage and opportunity to buy drugs may be moderated by illegal drug use. Both active illegal drug users and non-users living in the most disadvantaged neighbourhoods were more likely to be approached by a dealer.

With respect to female-male differences, males were more likely than females to have had recent drug purchase opportunities, as discussed previously. ${ }^{24}$ This sex difference was highly consistent across all types of neighbourhood disadvantage: both males and females in disadvantaged neighbourhoods were more likely to be approached by someone selling drugs than their counterparts residing in more advantaged neighbourhoods.

With respect to race-ethnicity, non-Hispanic black and Hispanic area residents are somewhat more likely to have experienced a recent drug purchase opportunity, as compared with their non-Hispanic white neighbours, but the excess odds actually are characteristics of males in these racialethnic minority groups of the population, and are not characteristic of females. Indeed, table 3 shows three remarkable estimates that merit future attention: (1) by comparison with non-Hispanic white females, the females of Asian, Pacific Islander, and other non-black, non-Hispanic groups are generally less likely to have experienced recent drug purchase opportunities; (2) the same type of inverse association is seen for non-black, non-Hispanic minority males living in the two most disadvantaged neighbourhoods; and (3) Hispanic men in the least disadvantaged neighbourhoods have a considerably greater chance to buy illegal drugs than other population subgroups, male and female. It might be best to check whether these results can be replicated in future surveys before anyone engages in detailed speculation about possible protective influences for Asian, Pacific Islander, and other non-Hispanic neighbourhood residents, or about the processes that differentiate Hispanic men living in the least disadvantaged neighbourhoods.

Despite the fact that the participants in this study come from a nationally representative sample, with data collected using standardised self report assessments, the cross sectional nature of the association merits attention. As mentioned in the introductory section of this article, this early study of drug purchase opportunities and neighbourhood environment has little resolving power with respect to underlying social causation and social selection processes that might lead to an observed association between levels of neighbourhood disadvantage and recent occurrence of drug purchase opportunities. For example, we cannot say that neighbourhood disadvantage leads to greater occurrence of drug purchase opportunities, or vice versa. And we cannot say whether people seeking drug purchase opportunities might move into neighbourhoods of greater social disadvantage (that is, in an effort to get closer to drug purchase opportunities associated with neighbourhood disadvantage). As with the reciprocal process models that posit density of drug purchase opportunity as a cause of later neighbourhood disadvantage, these are processes best investigated in a future line of longitudinal investigations to build upon the foundation of the current cross sectional data.

In addition, we again must acknowledge measurement complications. A limitation is the self report character of the data. It is possible, if not plausible, that the experience of being approached by a drug dealer near home yields a change in the evaluation of the local neighbourhood, and makes the local area residents more sensitive to other characteristics of disadvantage. A strength of this study is the use of multiple sources within the same community segments as raters of the neighbourhood environment, rather than relying on a person's own report. In future research, other approaches to strengthen the measurement of neighbourhood disadvantage can incorporate independently recorded data (for example, arrest rates).

Another limitation of this study is that the location of the purchase opportunity is unknown. Many drug users travel into other neighbourhoods to obtain their drugs, and the drug purchase opportunity may not necessarily be reflective of what happens in their own residential neighbourhoods. In addition, sales are not the only vehicle of transaction in a drug purchase opportunity. Youths may be approached and enticed by free "samples" or allowed to share in the experience of a smoke or a hit.

Another challenge for future research is to evaluate suspected pathways or mechanisms that might link neighbourhood contexts to having an increased opportunity and access to drugs. For example, neighbourhood may be associated with drug opportunities indirectly if there are increased social stressors and higher levels of psychological distress among residents in disadvantaged neighbourhoods. ${ }^{12}$ These psychological experiences may increase the likelihood of seeking drug sources, or may make a person with visible signs of social disengagement more likely to be approached by a drug dealer. ${ }^{1}$

\section{Conclusions}

Limitations notwithstanding, this study adds new evidence about the potential importance of neighbourhood disadvantage as a feature of environmental and psychosocial contexts that might help epidemiologists account for the non-random distribution of drug involvement in human populations. To develop effective community level interventions we must understand and seek to explain how various aspects of the social context (for example, neighbourhoods, schools, families, peers) work independently and jointly with one another. The evidence from this study suggests that teenage girls and adult women are less likely to be approached by someone selling drugs, no matter how disadvantaged the neighbourhood. If confirmed, this evidence of possible sex linked protection can be traced back to its sources (for example, different levels of parental monitoring for males compared with females, greater male involvement in 
neighbourhood activities that may be associated with higher risk for drug exposure opportunities, or different gender specific outlets for obtaining illegal drugs). With new insights it may be possible to develop new methods to prevent illegal drug use across a range of social disadvantage. Programmes that target drug purchase opportunities while also changing the environment that supports the drug context might break cycles of drug involvement, as well as active drug seeking behaviour, which otherwise perpetuate drug associated public health hazards.

\section{ACKNOWLEDGEMENTS}

Data reported herein come from national survey data collected under the auspices of the Office of Applied Studies, Substance Abuse and Mental Health Services Administration.

\section{Authors' affiliations}

C L Storr, C-Y Chen, J C Anthony, Department of Mental Health, Johns Hopkins Bloomberg School of Public Health, Baltimore, USA

J C Anthony, Department of Epidemiology, Johns Hopkins Bloomberg School of Public Health

Funding: the work was supported by NIDA R01DA09897.

Conflicts of interest: none declared.

\section{REFERENCES}

1 Rosenberg MF, Anthony JC. Aggressive behavior and opportunities to purchase drugs. Drug Alcohol Depend 2001;63:245-52.

2 James KE, Wagner FA, Anthony JC. Regional variation in drug purchase opportunity among youths in the United States, 1996-1997. J Urban Health 2002;79:104-12.

3 Anthony JC, Neumark TD, Van Etten ML. Do I do what I say? A perspective on self-report methods in drug dependence epidemiology. In: Stone AA, Turkon JS, Bachrach CA, et al, eds. The science of self-report implications for research and practice. Mahwah, NJ: Lawrence Erlbaum Associates, 2000:175-98.

4 O'Donnell JJ, Voss HL, Clayton RR, et al. Young men and drugs- a nationwide survey. NIDA Res Monogr 1976;5:1-144.

5 Van Etten ML, Anthony JC. Initial opportunity to use marijuana and the transition to first use: United States, 1979-1994. Drug Alcohol Depend 1997:49:1-7.
6 Van Etten ML, Anthony JC. Male-female differences in transitions from first drug opportunity to first use: searching for subgroup variation by age, race, region, and urban status. J Womens Health 2001;10:797-804.

7 Wagner FA, Anthony JC. Into the world of illegal drug use: exposure opportunity and other mechanisms linking the use of alcohol, tobacco, marijuana, and cocaine. Am J Epidemiol 2002;155:918-25.

8 Crum RM, Lillie-Blanton M, Anthony JC. Neighborhood environment and opportunity to use cocaine and other drugs in late childhood and early adolescence. Drug Alcohol Depend 1996:43:155-61.

9 Storr CL, Arria AM, Workman R, et al. Neighborhood environment and opportunity to try methamphetamine ('ice') and marijuana: evidence from Guam. Subst Use Misuse (in press).

10 Ensminger ME, Anthony JC, McCord J. The inner city and drug use: initial findings from an epidemiological study. Drug Alcohol Depend 1997;48:175-84.

11 Saxe L, Kudushin C, Beveridge A, et al. The visibility of illicit drugs: implications for community-based drug control strategies. Am J Public Health 2001:91:1987-94.

12 Boardman JD, Finch BK, Ellison CG, et al. Neighborhood disadvantage, stress, and drug use among adults. J Health Soc Behav 2001;42:151-65.

13 Sampson RJ, Raudenbush SW, Earls F. Neighborhoods and violent crime: a multilevel study of collective efficacy. Science 1997;277:918-24.

14 Wilson JQ, Kelling GL. Broken Windows. In: Dunham RG, Alpert GP, eds. Critical issues in policing: contemporary readings. Prospect Heights, IL: Waveland Press, 1989.

15 Reno R, Cialdini RB, Kallgren CA. The trans-situational influence of social norms. J Pers Soc Psychol 1993;64:104-12.

16 Cohen D, Spear S, Scribner R, et al. "Broken windows" and the risk of gonorrhea. Am J Public Health 2000;90:230-6.

17 Speer PW, Gorman DM, Labouvie EW, et al. Violent crime and alcohol availability in an urban community. J Public Health Policy 1998;19:303-18.

18 Dohrenwend BP, Levav I, Shrout PE, et al. Socioeconomic status and psychiatric disorders: the causation-selection issue. Science 1992;21:946-52.

19 Petronis KR, Anthony JC. A different kind of contextual effect: geographical clustering of cocaine incidence in the USA. J Epidemiol Community Health 2003; 57:893-900.

20 Petronis KR, Anthony JC. Perceived risk of cocaine use and experience with cocaine: do they cluster within US neighborhoods and cities? Drug Alcohol Depend 2000;57:183-92

21 Xiaoming L, Stanton B, Feigelman S. Exposure to drug trafficking among urban, low-income African American children and adolescents. Arch Pediatr Adolesc Med 1999;153:161-8.

22 US Substance Abuse and Mental Health Service Administration. National Household Survey on drug abuse main findings 1998. Rockville, MD: United States Department of Health and Human Services, 2000.

23 Nunnally JC. Psychometric theory. 2nd edn. New York: McGraw-Hill, 1978.

24 Van Etten ML, Neumark YD, Anthony JC. Male-female differences in the earliest stages of drug involvement. Addiction 1999;94:1413-19. 